\title{
The role of RCP chief registrars in the COVID-19 pandemic response
}

\author{
Authors: Victoria Gaunt, ${ }^{\mathrm{A}}$ Georgia Sayer, ${ }^{\mathrm{A}}$ Jacqueline Bassett ${ }^{\mathrm{A}}$ and Lucy Ellis ${ }^{\mathrm{A}}$
}

\section{Introduction}

Gloucestershire Hospitals NHS Foundation Trust (GHNHSFT) was an early adopter of the Royal College of Physicians (RCP) Chief Registrar Programme and has four chief registrars (two in medicine and two in emergency medicine).

The COVID-19 pandemic created an unprecedented requirement for process and protocol change. The chief registrar role evolved as the pandemic did, providing numerous opportunities but also unique challenges.

The first COVID-19 case confirmed at the trust was on 14 March 2020, and to date 874 positive cases have been seen at the trust, the highest level of COVID-19 activity in the south west of England.

\section{Materials and methods}

The GHNHSFT chief registrars led numerous projects during this period falling into the following areas: pathways and reconfiguration, communication, staffing, education and wellbeing.

The chief registrars gained rapid experience in writing documentation including the trust COVID-19 cardiac arrest algorithm, the ambulatory care pathway and the COVID-19 clerking pro forma.

The chief registrars ran a biweekly junior doctor teleconference following an agenda that covered items such as: process changes due to COVID-19, personal protective equipment (PPE) guidance reminders, signposting to wellbeing resources and opportunity to ask questions.

They wrote and organised the medicine and emergency department (ED) junior doctor rotas for the duration of the pandemic.

The chief registrars played a role in junior doctor education, running COVID-19 in situ simulations in the ED, setting up ED middle-grade virtual teaching and inducting redeployed doctors to the ED and medicine.

Initiatives were introduced by the chief registrars to promote junior doctor wellbeing such as pre-and post-shift wellness checks and an ED doctor buddy system.

Author: ${ }^{A}$ Gloucestershire Hospitals NHS Foundation Trust, UK

\section{Results and discussion}

Working during the pandemic presented a unique learning opportunity to put into practice the theory learnt at the RCP chief registrar study days by leading multiple projects with rapid implementation of change. They were able to learn from the styles of senior leaders at the trust, while acting as a conduit for them to gauge junior doctor opinions and circulate information.

They received overwhelming positive feedback. Senior leaders commented that they provided 'clear and visible leadership to junior doctors at a time of huge change and pressure' while junior doctors commented 'our chief registrars cannot receive enough thanks on my behalf for the amount of hard work they provided in ensuring medical leadership for our rotas'.

Learning to lead through a crisis has accelerated development of their leadership skills and they have recognised the significance of key qualities such as remaining calm and visible, leading by example, listening to the concerns of every individual and never underestimating the importance of communication.

\section{Conclusion}

Chief registrars were integral to the successful pandemic response at GHNHSFT. Having four chief registrars allowed a considerable variety of projects and schemes to be implemented rapidly, while the role as a bridge between junior doctors and senior management to disseminate vital communication and training was essential. It further highlights how the unique role of the chief registrar can help doctors to develop as leaders while providing a considerable benefit to the hospital trust.

\section{Conflicts of interest}

None declared. 